\title{
Mass Transfer Prediction of Gas-Liquid Contacting in a Rotating Spiral Channel
}

\author{
Ahmed A. Ayash, Jordan M. MacInnes \\ University of Sheffield, Department of Chemical and Biological Engineering \\ Mapping Street, Sheffield S1 3JD, UK \\ Ahmedayash@sheffield.uk.ac; j.m.macinnes@sheffield.ac.uk
}

\begin{abstract}
Numerical solution of the governing equations for mass, momentum and species can be used to predict mass transfer in a rotating spiral device. The case of a dilute solute transferring in counter-current gas-liquid flow is considered. Computations in a twodimensional section of the flow with an existing model for interface shape are used to determine the velocity and solute species fields in each phase. The prediction is assessed along with that of an existing analytical solution for infinite channel width by comparison with some recent mass transfer coefficient data for acetone desorbing from water into air over a range of water flow rates. The computation reproduces the measured results well over the full range of the data. At higher liquid flow rates it is found that secondary motion in each phase generated by Coriolis acceleration acting on the gas phase, causes a doubling of mass transfer coefficient.
\end{abstract}

Keywords: rotating spiral channel; mass transfer coefficient; Coriolis secondary motions; interface shape

\section{Introduction}

Molecular separations involving gas-liquid contacting are the backbone of many chemical processes and include absorption, stripping and distillation, but equally many arise in analytical applications. The rotating spiral technique is an approach having the potential to produce controlled contact between any two immiscible fluid phases [1]. It rests on the principle of guiding a gas and a liquid to flow in a continuous path without the phases mixing (Fig. 1). The technique uses a spiral channel spinning around an axis through its origin to produce both centrifugal and Coriolis acceleration. The centrifugal acceleration with adjustment of the pressure gradient along the channel allows the fluid phases to flow either counter-currently or co-currently, side by side as two separate layers. The Coriolis acceleration and spatial variation of streamwise velocity produce secondary motion that can enhance mass transfer. The rotating spiral allows simultaneous control of the phase layer thicknesses and the phase flow rates, consequently allowing optimum contacting regardless of phase and transferring solute properties. Thus, it opens up the possibility of handling fluid phase systems having widely differing viscosities, densities, solute equilibrium characteristics and solute diffusivities, and avoids limitations inherent in existing techniques where phase mixing occurs.

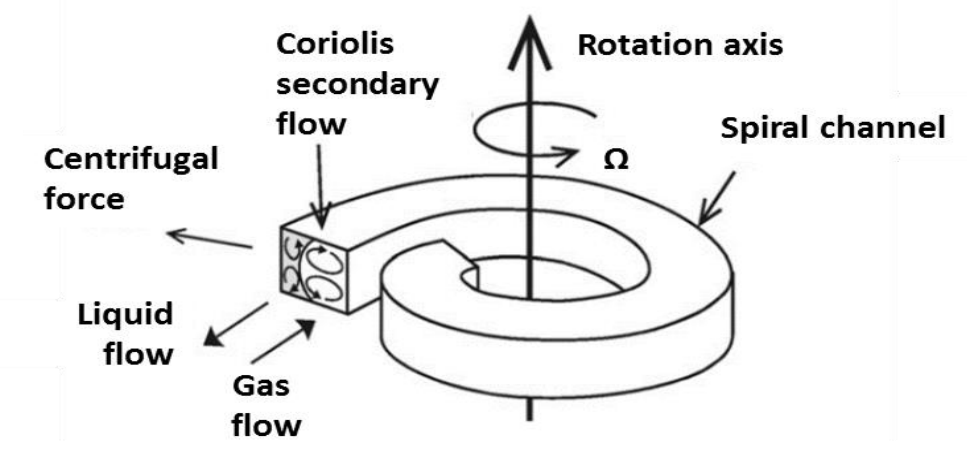

Fig. 1: Two immiscible phases contacting in a rotating spiral channel. 
An important feature of rotating spiral contacting flow is that full theoretical treatment is possible, opening the way to direct prediction of design performance. Ortiz-Osorio et al. [2] computed the velocity and species distributions in a 2-D section of a spiral channel in a successful comparison with the experimental result of MacInnes et al. [3]. The shape of the interface can be determined independently of the flow and mass transfer since the transverse component of centrifugal acceleration that controls interface shape is far greater than that along the channel that determines the flow stresses [1]. This decouples the solution for velocity and species from the determination of the geometry of the phase regions.

Here, this computational approach will be tested using data from an experimental study that is currently underway of mass transfer in a rotating spiral device. The device is that used in MacInnes and Zambri [1] where detailed measurements were made of liquid layer thickness over a range of gas and liquid flow rates for air-water contacting. That work also includes details of the model used here for phase interface shape. It is a relatively simple matter to add a transferring solute to the water and thereby make determinations of mass transfer coefficient in the same device and, indeed, measurements for various organic solutes representing a range of phase equilibrium characteristics have now been made. In the study here, data for acetone over a range of liquid flow rate, from that work, are used to provide a preliminary assessment of the 2-D computation. The conditions in the spiral channel in the experiments are $24{ }^{\circ} \mathrm{C}$ and 1.8 bar and the acetone remains in dilute concentration (less than $2 \%$ by mole in each phase). These conditions give an estimated equilibrium ratio of acetone mole fraction in the gas and liquid phases of $f^{\prime}=1.15$. The rotation rate used is $3200 \mathrm{rpm}$.

Other work has pursued analytical solutions for infinite channel width, where the description requires just one spatial dimension. Tilley et al. [4] and others provide the solution for velocity profiles in both phases and this is followed in MacInnes et al. [5] with the solution for species profiles in both phases. These analytical solutions are used here to provide the convenient limiting reference case of contacting in an infinitely wide channel, a situation where no secondary motion results from Coriolis acceleration.

\section{Model}

The governing equations can be expressed for the two-phase flow in a rotating spiral channel using the coordinate system and the geometric parameters defined in Fig. 2.

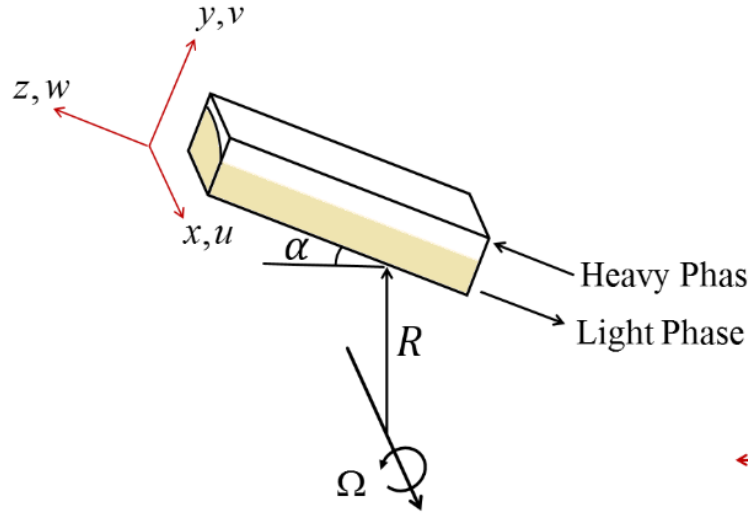

(a) Channel Segment

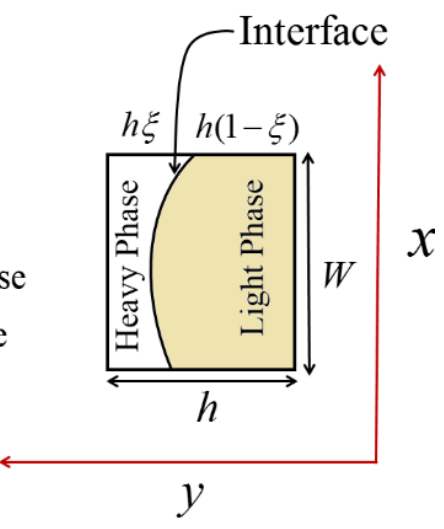

(b) Channel Section

Fig. 2: Channel geometry: (a) segment of a spiral channel and (b) the 2-D section.

Since practical spiral channels have a relatively large radius of curvature, i.e. $R>>h$, the channel is treated as straight at any location but with angle $\alpha$ relative to the tangential direction. Typically, the rotating spiral channel operates with flow in the laminar regime, the entry lengths are short and the gas can be treated as incompressible. Also, for dilute solute concentration, beyond the entry regions the flow in each phase is fully developed so that the pressure gradient in the streamwise $(z)$ direction is constant and equal to the bulk value, $d P_{B} / d z$. Further, the change in solute concentration in the $z$ direction can be replaced approximately by the change in the bulk value, $d Y_{B} / d z$, since the concentration distribution in the $x-y$ plane varies slowly in that direction. Under these conditions and using the notation shown in Fig. 2 , the governing equations for the three velocity components ( $u, v$, and $w$ in $x, y$, and $z$, respectively), the solute mole 
fraction $(Y)$ and the piezometric pressure $(p)$ may be written in each phase region (in terms of the appropriate values for mass density, $\rho$, molar density, $n$, viscosity, $\mu$, and diffusion coefficient, $D$ ) as:

Continuity Equation:

$$
\frac{\partial u}{\partial x}+\frac{\partial v}{\partial y}=0
$$

Momentum Equations:

$$
\begin{aligned}
& \rho u \frac{\partial u}{\partial x}+\rho v \frac{\partial u}{\partial y}=-\frac{\partial p}{\partial x}+\mu\left(\frac{\partial^{2} u}{\partial x^{2}}+\frac{\partial^{2} u}{\partial y^{2}}\right) \\
& \rho u \frac{\partial v}{\partial x}+\rho v \frac{\partial v}{\partial y}=-\frac{\partial p}{\partial y}+\mu\left(\frac{\partial^{2} v}{\partial x^{2}}+\frac{\partial^{2} v}{\partial y^{2}}\right)+\underbrace{2 \rho \Omega w}_{\text {Coriolis term }} \\
& \rho u \frac{\partial w}{\partial x}+\rho v \frac{\partial w}{\partial y}=-\frac{d P_{B}}{d z}+\mu\left(\frac{\partial^{2} w}{\partial x^{2}}+\frac{\partial^{2} w}{\partial y^{2}}\right)+\underbrace{\rho R \Omega^{2} \sin \alpha}_{\text {Centrifugal term }}-\underbrace{2 \rho \Omega v}_{\text {Coriolis term }}
\end{aligned}
$$

Species Equation:

$$
n u \frac{\partial Y}{\partial x}+n v \frac{\partial Y}{\partial y}=-n w \frac{d Y_{B}}{d z}+n D\left(\frac{\partial^{2} Y}{\partial x^{2}}+\frac{\partial^{2} Y}{\partial y^{2}}\right)
$$

These equations apply to a 2-D section of the channel ( $x-y$ plane) at any particular position along the spiral. The equations are modified for the rotating reference frame by the appearance of a centrifugal term in the streamwise momentum equation (Eq. 4) and Coriolis terms in both the transverse and streamwise momentum equations (Eqs. 3 and 4). It is the Coriolis term in the transverse momentum equation that produces the secondary motions depicted in Fig. 1. The boundary conditions at the fixed and non-adsorbing walls are no slip for velocity and zero flux for species. At the phase interface the further conditions of continuity of velocity and balance of stresses must be satisfied. Details of these are given in Ortiz-Osorio et al. [2]. Also, the species mole fraction is required to satisfy equilibrium at the interface, so $Y_{V}=f^{\prime} Y_{L}$ where subscripts ' $V$ ' and ' $L$ ' signify gas and liquid values, respectively, and $f^{\prime}$ ' is the slope of the equilibrium curve at dilute solute concentration.

The region of the solution domain occupied by each separate phase (having areas $A_{V}$ and $A_{L}$ ) must be established to solve the governing equations. MacInnes and Zambri [1] point out that for practical conditions the component of centrifugal acceleration driving the flows is far smaller than the transverse component that determines interface shape, i.e. $R \Omega^{2} \sin \alpha<<R \Omega^{2} \cos \alpha$, since $\alpha$ is small. This means that the interface shape is not significantly affected by flow stresses. Taking advantage of small angle approximations and with gravity in the $x$ direction for the devices considered, interface shape is determined by the Laplace equation relating local interface radius of curvature, $r$, to interfacial surface tension, $\sigma$, radial position, $R$, the densities of the phases and centrifugal and gravitational accelerations [1]:

$$
\frac{\sigma}{r}=\Delta p_{o}-\left(\rho_{L}-\rho_{V}\right)\left(R \Omega^{2} y+g x\right)
$$

$\Delta p_{o}$ is the pressure difference across the interface at position $(x, y)=(0,0)$.

It is also necessary to specify the contact angles where the side walls intercept the interface. The increasing radial position $(R)$ with distance along a spiral channel means that the solution to Eq. 6 and hence interface shape varies along the channel. The effect is relatively modest and the interface shape used here is calculated using an intermediate radial position, specifically the average of the radial positions at the inner and outer ends of the spiral. 


\section{Analysis}

The mass transfer coefficient is the basis of comparison between the experiment and the computation. In the experiment, measurement of volume flow rate, $Q$, and inlet and outlet solute mole fractions in each phase allows the overall mass transfer coefficient to be determined, provided the flow rate remains unchanged and mass transfer coefficients are constant along the contacting length from one end to the other. As indicated, the interface shape changes somewhat with distance along the channel as a result of the changing radial position and hence centrifugal acceleration, so the resulting mass transfer coefficient is an effective one. This overall mass transfer coefficient in terms of liquid phase quantities, $K_{L}$ is determined from the measured quantities using the standard relation (subscripts ' $L$ ', ' $V$ ' and ' $B$ ' designate liquid phase, gas phase and bulk quantities, respectively):

$$
K_{L}=\frac{n_{L} Q_{L}}{W L} \frac{\left\lfloor\left(Y_{L B}\right)_{I N}-Y_{L B}\left(Y_{L B}\right)_{O U T}\right\rfloor}{\overline{\Delta Y_{L B}}}
$$

Where $\overline{\Delta Y_{L B}}=\frac{\left(\Delta Y_{L B}\right)_{I N}-\left(\Delta Y_{L B}\right)_{O U T}}{\ln \left[\left(\Delta Y_{L B}\right)_{I N} /\left(\Delta Y_{L B}\right)_{O U T}\right]}$ with $\Delta Y_{L B}=Y_{L B}-Y_{L B}^{*} \quad$ and $\quad Y_{L B}^{*}=Y_{V B} / f^{\prime}$

Arriving at Eq. 7 uses the approximation $a_{p}=1 / h$ for interface area per passage volume.

From the 2-D computations, it is possible to determine the mass transfer coefficients individually $\left(k_{V}\right.$ and $\left.k_{L}\right)$ using the definitions for these:

$$
k_{V}\left(Y_{V B}-Y_{V I}\right)=-n_{V} w_{V B} \frac{d Y_{V B}}{d z} \frac{A_{V}}{W} \quad k_{L}\left(Y_{L I}-Y_{L B}\right)=n_{L} w_{L B} \frac{d Y_{L B}}{d z} \frac{A_{L}}{W}
$$

$A_{V}$ and $A_{L}$ are the section areas occupied by the gas and liquid phases and the bulk values are determined from a given computed solution using the definitions,

$$
\begin{array}{cc}
w_{V B}=\frac{1}{A_{V}} \int_{A_{V}} w_{V} d A & w_{L B}=\frac{1}{A_{L}} \int_{A_{L}} w_{L} d A \\
Y_{V B}=\frac{1}{w_{V B} A_{V}} \int_{A_{V}} w_{V} Y_{V} d A & Y_{L B}=\frac{1}{w_{L B} A_{L}} \int_{A_{L}} w_{L} Y_{L} d A
\end{array}
$$

Subscript ' $I$ ' in Eqs. 8 indicates average value over the interface length, $\ell_{I}$, sliced by the domain section,

$$
Y_{V I}=\frac{1}{\ell_{I}} \int_{\ell_{I}} Y_{V} d \ell \quad Y_{L I}=\frac{1}{\ell_{I}} \int_{\ell_{I}} Y_{L} d \ell
$$

Since the equilibrium relation is linear and the normal solute fluxes on each side of the interface are equal, the overall mass transfer coefficient can be expressed in terms of the individual gas and liquid mass transfer coefficients as:

$$
K_{L}=\left(\frac{1}{f^{\prime} k_{V}}+\frac{1}{k_{L}}\right)^{-1}
$$




\section{Numerical Solution}

It is clear that solution of the governing equations requires specification of the bulk pressure gradient $\left(d P_{B} / d z\right)$, the bulk solute mole fraction gradient $\left(d Y_{B} / d z\right)$ and the rotation rate $(\Omega)$ along with the physical properties of each phase and the geometric parameters, including the position of the interface in the domain section. The properties are different in each phase region and the values used (Table 1) are those of the two pure phases, air and water, at the spiral temperature and pressure. While the pressure gradient is the same in each phase (developed flow), the bulk mole fraction gradient is not, although the two are related by solute conservation (equating the right hand sides of Eqs. 8), so specification of just a single bulk mole fraction gradient is required. Further, since only gradients of pressure and mole fraction appear in the equations, it is necessary to specify the level of each at some position in the section.

Table 1: Phase and solute properties at $24^{\circ} \mathrm{C}$ and 1.8 bar.

\begin{tabular}{|c|c|c|c|}
\hline & $\rho\left(\mathrm{kg} / \mathrm{m}^{3}\right)$ & $\mu(\mathrm{Pa} \mathrm{s})$ & $D\left(\mathrm{~m}^{2} / \mathrm{s}\right)$ \\
\hline Gas phase & 2.1 & $1.86 \times 10^{-5} \quad[6]$ & $6 \times 10^{-6} \quad[6]$ \\
\hline Liquid phase & $986.4[7]$ & $1 \times 10^{-3}[8]$ & $1.24 \times 10^{-9} \quad[9]$ \\
\hline
\end{tabular}

Obtaining a solution directly for given volume flow rates is not possible. To achieve the solution for particular values of the phase flow rates requires trial and error adjustment of the interface position and the bulk pressure gradient. The experiments which will be predicted here are at a single gas flow rate but over a range of liquid flow rates. To predict what happens in the experimental conditions it is only necessary to match the gas flow rate and compute a number of different liquid flow rates. Thus, for a given interface position the bulk pressure gradient is adjusted to match the experimental gas flow rate with the liquid flow rate determined from the solution.

Setting the bulk mole fraction gradient and the value for solute mole fraction at a reference position in the section determine (together with the flow solution) the mole fraction fields in both phases and hence the bulk mole fraction in each phase in the computed section. Ortiz-Osorio et al. [2] found the computed mass transfer coefficients were insensitive to bulk mole fraction gradient but depended strongly on bulk mole fraction level for a channel with small aspect ratio $(W / h<1)$, while MacInnes et al. [5] show that mass transfer coefficients are entirely independent of both the bulk value and the gradient for infinite aspect ratio. Explorations of the effect of these for the intermediate aspect ratio of the experimental channel suggest a negligible effect of the choice of bulk value and gradient, so which particular values are used is only constrained by a selection that avoids the value of mole fraction falling outside the physically-possible range of zero to unity at all positions in the section. Since the mass transfer coefficients do not depend significantly on the local value and gradient of bulk mole fraction, it follows that the mass transfer coefficients are closely constant along the channel and it is unnecessary to compute mass transfer coefficients as a function of position along the channel. (The approximation associated with the variation of interface shape as radial position and hence centrifugal acceleration changes along the channel remains.)

The equations are solved numerically using Comsol Multiphysics, V5.2 with a grid of about 1400 and 4200 triangular elements respectively in the liquid and gas regions of the domain section. First-order Lagrange elements are used for pressure and second-order Lagrange elements for the velocity components and species. Doubling the number of elements produced less than a few percent change in the computed mass transfer coefficients. The physical properties used in the computations are those in Table 1 and correspond to the conditions of the experiments for solute-free air and a $2 \%$ acetone solution with water, i.e. the condition at the inlet for each phase. The required properties are estimated using a combination of standard empirical and theoretical relations and data from the literature (specific references given in the table). The solution domain is $1.5 \mathrm{~mm}$ high $(h)$ and $4 \mathrm{~mm}$ wide $(W)$, corresponding to the experimental channel dimensions. (The experimental channel also has length $L=900 \mathrm{~mm}$.) The only other input parameters required for the computation are the experimental spiral rate $R \sin \alpha=5.57 \times 10^{-4} \mathrm{~m}$ and rotation rate $\Omega=335 \mathrm{rad} / \mathrm{s}(3200 \mathrm{rpm})$. 


\section{Results}

Fig. 4 shows representative results from a computation which serve to highlight typical characteristics of rotating spiral contacting flow. On the left is the domain geometry with the dividing interface determined from solution of Eq. 6. The thickness of the liquid layer shown is about $75 \mu \mathrm{m}$ at the minimum point. The interface is asymmetric both since gravity causes slight tilting and different end wall materials (plastic at the top wall and glass at the bottom wall in the experiment) give different contact angles and hence meniscus heights. The thinness of the liquid layer in relation to that of the gas layer is typical for effective contacting [5]. The streamwise velocity contour plot shows clear distortion in the gas phase from pure pressure flow with relatively thin momentum layers at the two end walls and a relatively thick momentum layer along the interface. These distortions are caused by the Coriolis term in the $w_{V}$ equation, which serves to drive the gas flow where $v_{V}$ is positive and retard the gas flow when negative ( $\Omega$ is positive in the computation and experiment). The direction and magnitude of $v_{V}$ in each region of the flow can be seen qualitatively from the velocity vector plot showing motion in the plane of the section. The relationship between thin and thick momentum layers and the sign and magnitude of $v_{V}$ is apparent. The motions in the plane of the domain are caused by the Coriolis term in the $v_{V}$ equation which depends on the streamwise component of velocity and hence is strong in the central region of each phase and weak near the solid wall surfaces. This variation in strength produces the secondary circulating motions that are evident in the vector plot. Finally, the distribution of solute in each phase is clearly affected by both the secondary and the main motion of the fluids. The link between the patterns of variation in solute mole fraction in each phase and the secondary motion in each is immediately clear. The yellow core in the meniscus region of the liquid layer near the bottom end wall is a consequence of the larger streamwise velocity there producing stronger convection of liquid from upstream with high solute mole fraction. It can be noted that while the secondary motion affects the entire width of the flow in the gas phase, it only penetrates into the liquid from the end walls a distance similar to the liquid layer thickness. Hence, the influence of the secondary motion on mass transfer in the liquid phase may be expected to be relatively minor since only the very end regions of the flow are affected.
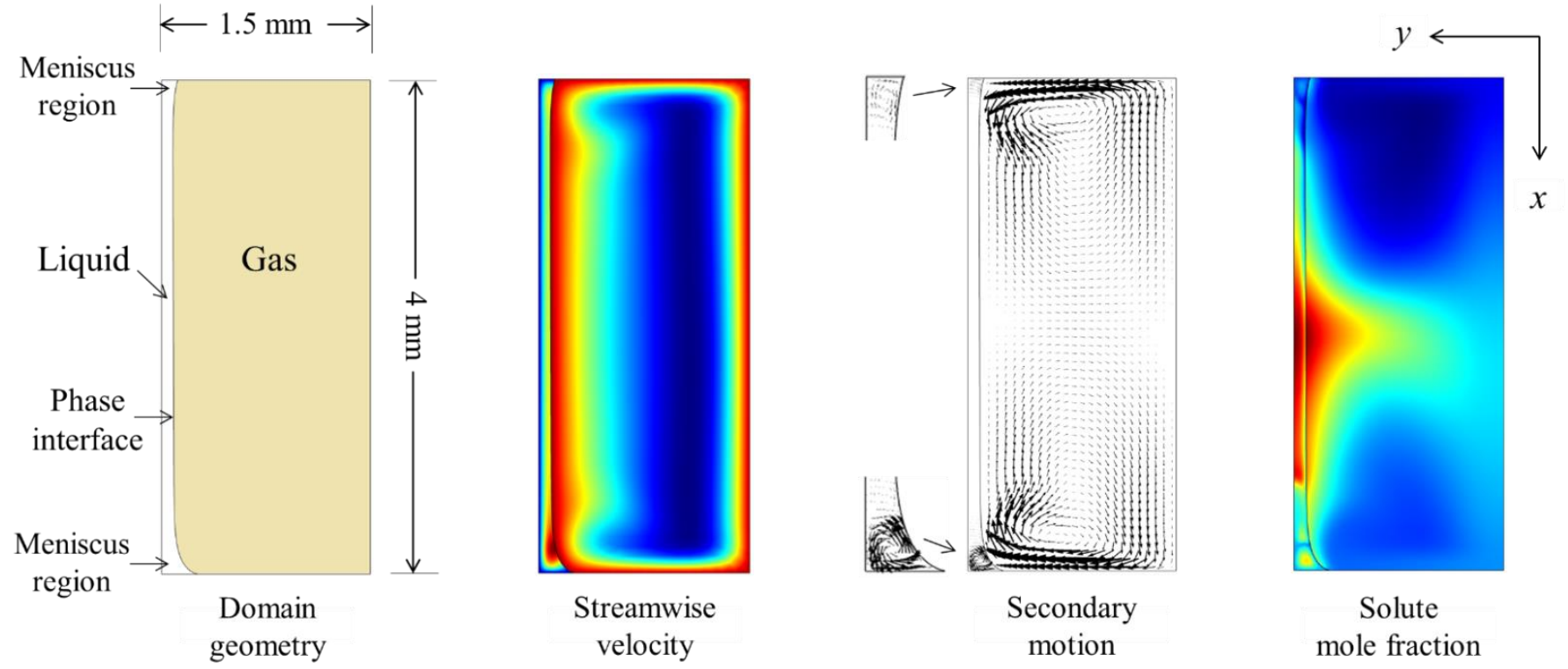

Fig. 4: Numerical results for $Q_{V}=3 \mathrm{NL} / \mathrm{min}$ and $Q_{L}=2.9 \mathrm{~mL} / \mathrm{min}$ at $3200 \mathrm{rpm}$ : domain geometry, streamwise velocity contours, vector plot revealing the secondary motion (arrow length proportional to magnitude in the plane of the section for each phase) and solute mole fraction contours.

The conditions used for the computation in Fig. 4 correspond to those in a series of experiments with acetone desorption in which different values of liquid flow rate were tested at the same gas flow rate and rotation rate. The mass transfer coefficient calculated for a series of computations spanning the experimental range of liquid flow rate are compared with the experimental results in Fig. 5. The experimental points are shown as solid diamonds while those for the 
2-D computation are shown with open squares. For reference, the corresponding result for infinite channel width (calculated from the analytical solution) is included as a dashed line. The other open symbols explore the contribution of various factors to the 2-D computation result.

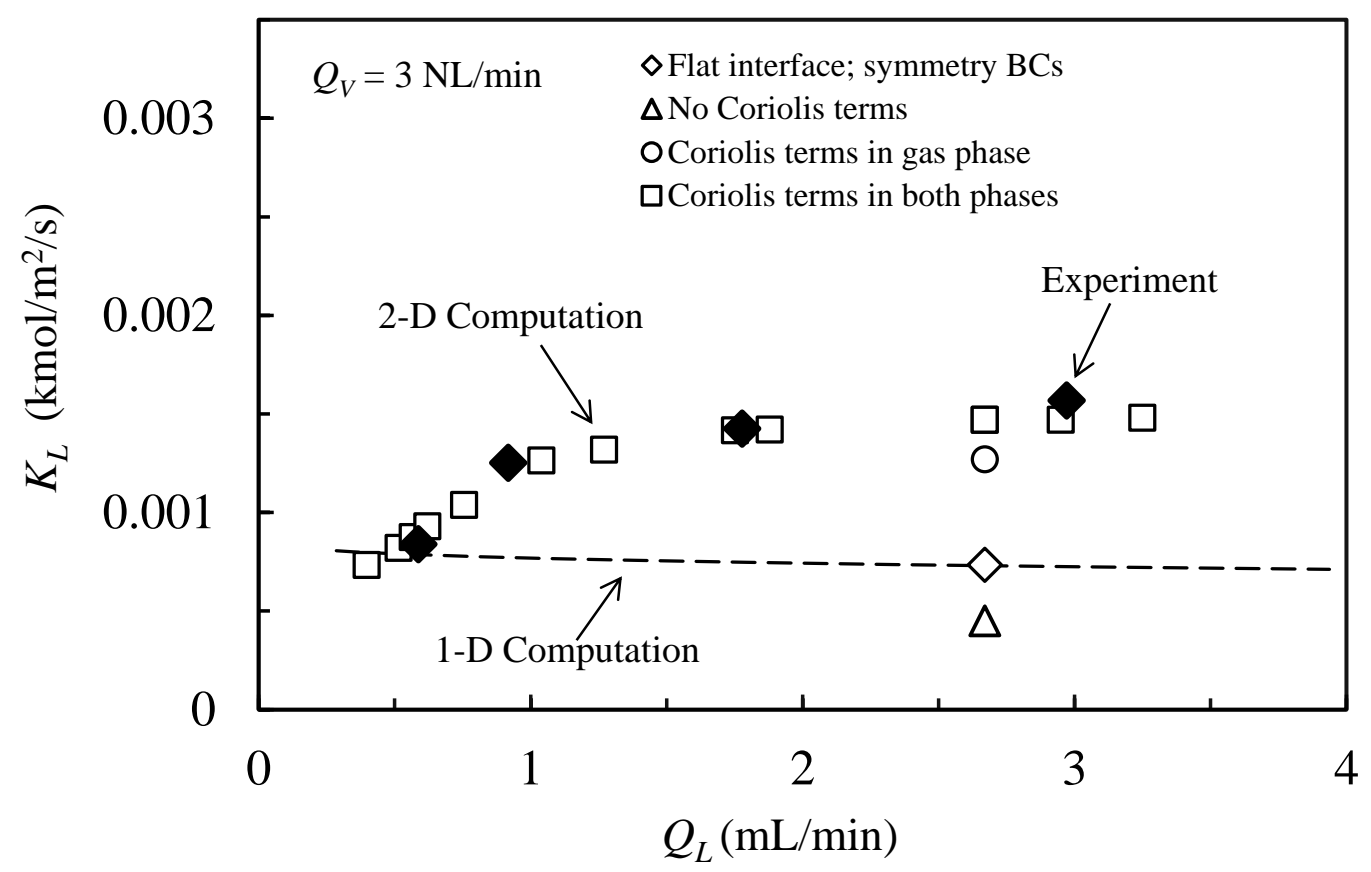

Fig. 5: Comparison of measured and predicted overall mass transfer coefficient over a range of liquid flow rate with fixed gas flow rate and rotation rate. Open squares are the 2-D computation and solid diamonds are the experimental measurements. The infinite-width channel result is shown as a dashed curve.

The computation closely predicts what is found experimentally and it is likely that the minor deviations seen are largely the result of experimental uncertainties. The results show that as $Q_{L}$ increases so initially does the mass transfer coefficient relative to the infinite-width result, until a plateau is reached where the overall mass transfer coefficient is approximately double that for an infinite-width channel. Since in the infinite-width channel the Coriolis term is balanced by hydrostatic pressure with no secondary motion generated, it is natural to suspect that secondary motion is what causes the doubling of mass transfer coefficient. At lower liquid flow rate (below about $1 \mathrm{~mL} / \mathrm{min}$ ) the decreasing liquid layer thickness presumably reduces the Reynolds number of the liquid flow to the extent that the secondary motions are significantly attenuated by viscosity.

The computation can be used to determine the role of the secondary motion and other factors in the mass transfer enhancement. First, the computation can be checked by reproducing the infinite-width case. This is done by imposing a flat interface and introducing symmetry boundary conditions at each end wall. The result at $Q_{L}=2.67 \mathrm{~mL} / \mathrm{min}$ is shown as an open diamond and is clearly in agreement with the analytical solution. Reintroducing the end wall boundary conditions and the correct interface shape for the actual channel, but leaving out the Coriolis terms and hence the secondary motion, leads to the result at the same liquid flow rate shown as an open triangle. Clearly, the effect of the end wall and curving interface is, overall, to reduce the mass transfer coefficient significantly. This reduction suggests that the enlarged space for liquid flow in the regions of the menisci, which should tend to reduce mass transfer by increasing both the characteristic streamwise velocity and diffusion length, overcome the opposite tendency from the end walls slowing the liquid near the menisci and the thinning of the central section of the liquid layer as liquid flow is diverted by the enlarged menisci regions. Next, it proves interesting to add the Coriolis terms back into the equations one step at a time, first putting them back into the gas phase equations (open circle) and then finally into the liquid phase equations, taking the computation back to the original result at that liquid flow rate (open square). Interestingly, the Coriolis terms in the gas 
phase alone bring the mass transfer coefficient up to nearly the original level, indicating that the direct effect of Coriolis terms in the liquid equations is of minor importance. The liquid secondary motion is apparently largely driven by the gas flow through shear stress at the phase interface. This view is consistent also with the attenuation of mass transfer occurring at low liquid flow rate. The gas flow rate and hence shear stress has not changed, but with the thinner liquid layer it is balanced by reduced secondary convective velocity.

\section{Conclusion}

Mass transfer coefficients determined experimentally for desorption of acetone from water-air system in a rotating spiral channel are used to assess the accuracy of 2-D numerical solution of the governing equations for flow and species in the case of a dilute solute. The results show that the 2-D computation convincingly captures the mass transfer performance over the range of the experimental data, the differences attributable to experimental uncertainties. It is shown that secondary motion resulting from Coriolis acceleration leads to a doubling of the mass transfer coefficient, with secondary motion in the liquid phase driven indirectly by the Coriolis secondary motion imparted to the gas phase. This preliminary work suggests that the 2-D computation can be an effective tool for identifying optimum channel designs for any particular application of a rotating spiral to gas-liquid contacting. However, a detailed assessment over a wider range of contacting conditions is required to establish the effectiveness of this approach in general and this work is currently underway.

\section{Acknowledgements}

The authors would like to thank the Iraqi Government for an HCED Scholarship (D 113508) supporting AAA.

\section{References}

[1] J. M. MacInnes and M. K. S. Zambri, "Hydrodynamic characteristics of a rotating spiral fluid-phase contactor," Chem. Eng. Sci., vol. 126, pp. 427-439, 2015.

[2] J. Ortiz-Osorio, J. M. MacInnes, P. J. Jordan, G. H. Priestman and R. W. K. Allen, "Computation of Mass transfer in Rotating Spiral Microchannel Distillation," in Proceedings The Eighth World Congress of Chemical Engineering, Montreal, 2009.

[3] J. M. MacInnes, J. Ortiz-Osorio, P. J. Jordan, G. H. Priestman and R. W. K. Allen, "Experimental demonstration of rotating spiral microchannel distillation," Chem. Eng. J., vol. 159, no. 1-3, pp. 159-169, 2010.

[4] B. S. Tilley, S. H. Davis and S. G. Bankoff, "Linear stability theory of two-layer fluid flow in an inclined channel," Phys. Fluids, vol. 6, no. 12, pp. 3906-3922, 1994.

[5] J. M. MacInnes, M. J. Pitt, G. H. Priestman and R. W. K. Allen, "Analysis of two-phase contacting in a rotating spiral channel," Chem. Eng. Sci., vol. 69, pp. 304-315, 2012.

[6] B. E. Poling and J. M. Prausnitz, The Properties of Gases and Liquids, 5th ed. McGRAW-HILL, 2001.

[7] K. S. Howard and R. A. McAllister, "Densities of Liquid-Acetone-Water Solutions Up to Their Normal Boiling Points," A.1.Ch.E. J., vol. 3, no. 2, pp. 161-164, 1957.

[8] K. S. Howard and R. A. McAllister, "The Viscosity of Acetone-Water Solutions Up to Their Normal Boiling Points," A.1.Ch.E. J., vol. 4, no. 3, pp. 362-366, 1958.

[9] C. R. Wilke and P. Chang, "Correlation of Diffusion Coefficients in Dilute Solutions," A.I.Ch.E. J., vol. 1, no. 2, pp. 264-270, 1955. 